\title{
Estado nutricional, anemia y parasitosis intestinal en los niños y adolescentes del Hogar de Amor y Esperanza, Tegucigalpa, año 2017
}

Nutritional status, anemia and intestinal parasitosis in children and teenagers from Hogar de Amor y Esperanza, Tegucigalpa, year 2017

DOI 10.5377/rct.v0i24.7877

\author{
Renata Valle Suárez \\ Karin Milla García ${ }^{2}$ \\ Doris Chinchilla Ticas ${ }^{3}$ \\ Vania Molina Flores ${ }^{4}$
}

\section{RESUMEN}

Objetivo: Identificar el estado nutricional por antropometría, la presencia de anemia y parásitos de los niños, niñas y adolescentes del Hogar de Amor y Esperanza en la Colonia San José de la Vega de Tegucigalpa en 2017, para determinar alteraciones nutricionales y derivarlos a los servicios de salud. Método: Estudio con un enfoque cuantitativo, no experimental, descriptivo y transversal de la población total de edades entre 5 y 17 años. Resultados: Según el índice de masa corporal (IMC) se obtuvo que $0,85 \%$ presenta desnutrición severa, 0,85\% desnutrición moderada, $94,02 \%$ normal, y $4,27 \%$ sobrepeso. De acuerdo a la talla para la edad de los niños y niñas de 5 a 10 años $16,0 \%$ se encuentra por debajo del percentil 3, 26\% en percentil $3,48 \%$ en percentil $15,8 \%$ en percentil 50 y $2 \%$ en percentil 85 . De acuerdo al peso para la edad: $10,0 \%$ se encuentra por debajo del percentil $3,20 \%$ en percentil $3,34 \%$ en percentil $15,28 \%$ en percentil $50,6 \%$ en percentil 85 y $2 \%$ en percentil 97 . El $77,78 \%$ presentan un hemograma normal, 5,98\% anormal y en el porcentaje restante no se realizó esta prueba. Se encontró la presencia de parásitos protozoarios y metazoarios en $80.56 \%$, y las especies prevalentes fueron Blastocystis hominis, Endolimax nana quiste, Entamoeba coli quiste e lodamoeba Butschlii quiste. Se observó la

1 Profesora del Departamento de Control Químico, Facultad de Ciencias Químicas y Farmacia, UNAH: renata.valle@unah.edu.hn

2 Profesora del Departamento de Química, Facultad de Ciencias Químicas y Farmacia. UNAH: karin.milla@unah.edu.hn

3 Profesora del Departamento de Control Químico, Facultad de Ciencias Químicas y Farmacia, UNAH: doris.chinchilla@unah.edu.hn

${ }^{4}$ Profesora del Departamento de Química, Facultad de Ciencias Químicas y Farmacia. UNAH: vania.molina@unah.edu.hn 
presencia de 1 parásito en $27.78 \%$, presencia de 2 parásitos $31.94 \%$, presencia de 3 parásitos $20.83 \%$ y no se observaron parásitos en el $19.44 \%$ de los niños y adolescentes del HAYE.Conclusión: La población presenta buen estado nutricional de acuerdo a los parámetros de IMC, examen hematológico y de heces, encontrándose mayor prevalencia de retraso en la talla que en el peso.

Palabras clave: Estado nutricional, índice de masa corporal, anemia y parásitos.

\section{ABSTRACT}

Objective: To identify the nutritional status by anthropometry, the presence of anemia and parasites of children and adolescents of the Hogar de Amor y Esperanza in Colonia San José de la Vega of Tegucigalpa, year 2017, to determine nutritional alterations and refer them to services of health. Method: Quantitative and non-experimental study, with a descriptive and transversal approach of the total population between 5 and 17 years of age. Results: According to the body mass index (BMI), it was found that $0.85 \%$ presented severe malnutrition, $0.85 \%$ moderate malnutrition, $94.02 \%$ normal, and $4.27 \%$ were overweight. According to the average size for boys and girls between 5 to 10 years of age; $16.0 \%$ are below the 3th percentile, $26 \%$ in the 3th percentile, $48 \%$ in the 15 th percentile, $8 \%$ in the 50 th percentile and $2 \%$ in percentile 85 . According to weight regarding age: $10.0 \%$ are below the 3 th percentile, $20 \%$ in the 3 th percentile, $34 \%$ in the 15 th percentile, $28 \%$ in the 50 th percentile, $6 \%$ in the 85 th percentile and $2 \%$ in 97 th percentile. $77.78 \%$ had a normal blood cell count, $5.98 \%$ abnormal and in the remaining percentage this test was not performed. The presence of protozoan and metazoan parasites was found in $80.56 \%$, and the prevalent species were Blastocystis hominis, Endolimax nana cyst, Entamoeba coli cyst and lodamoeba Butschlii cyst. The presence of 1 parasite was observed in $27.78 \%$, presence of 2 parasites $31.94 \%$, presence of 3 parasites $20.83 \%$ and no parasites were observed in $19.44 \%$ of the children and adolescents of HAYE. Conclusion: The population presents good nutritional status according to the parameters of BMI, hematological examination and stool, finding a higher prevalence of delay in height than in weight.

Keywords: nutritional status, body mass index, anemia and parasites. 


\section{INTRODUCCIÓN}

La evaluación nutricional se basa en el estudio de la incidencia de la nutrición sobre la salud. La interpretación de las pruebas bioquímicas, las medidas antropométricas, los resultados de la exploración física, la historia clínica y los cuestionarios dietéticos forman el criterio más confiable para diagnosticar la desnutrición y obesidad, el efecto de la alimentación en la composición corporal, el metabolismo de los nutrientes y los procesos nutricionales o fisiopatológicos resultantes (Martínez y Portillo, 2011).

Uno de los métodos más fiables para valorar el crecimiento es el estudio antropométrico, es decir la aplicación de técnicas biomédicas que expresan cuantitativamente la forma del cuerpo, lo que recibe el nombre de antropometría. Los parámetros antropométricos fundamentales son los que miden el crecimiento en longitud, el aumento de masa corporal, el crecimiento cerebral y la composición corporal en especial el tejido magro y graso (FUNIBER, 2011).

UNICEF desde 1980, en su principal publicación Estado Mundial de la Infancia hace un análisis más amplio sobre las tendencias mundiales que afectan a la infancia. En el informe de UNICEF en Dublín el 2013 destaca que (UNICEF, 2013):

"Uno de cada cuatro niños y niñas menores de 5 años en todo el mundo sufre de retraso en el crecimiento debido a la desnutrición crónica en las etapas cruciales del crecimiento. Mejorar la nutrición infantil es el imperativo para el progreso mundial que es posible lograr confirmar. El retraso en el crecimiento infantil no consiste solamente en tener una baja estatura para la edad. También significa sufrir un retraso en el desarrollo del cerebro y de la capacidad cognoscitiva."

"El retraso en el crecimiento puede eliminar las oportunidades en la vida de un niño y eliminar las oportunidades para el desarrollo de una nación", dijo el Director Ejecutivo de UNICEF, Anthony Lake. Eliminar el retraso en el crecimiento y otras formas de desnutrición es factible y asequible (UNICEF, 2013).

La etapa del crecimiento es un proceso acelerado, en el que la nutrición y la actividad física son factores importantes que tienen una marcada influencia en el grado de adiposidad, dependiente del equilibrio entre la ingesta y el gasto energético (Mataix y López, 2009).

Existen grupos vulnerables para los cuales es imperativo realizar la evaluación nutri- 
cional, entre ellos los niños y adolescentes en la etapa de crecimiento ya que se incrementan sus necesidades nutricionales (Mataix y López, 2009).

La niñez y la adolescencia son etapas para adoptar hábitos saludables de alimentación y de actividad física que pueden favorecer al bienestar físico y sicológico promoviendo estilos de vida saludable para disminuir el desarrollo de enfermedades crónicas no transmisibles en la edad adulta (Mataix y López, 2009).

La evaluación del estado nutricional mediante las medidas antropométricas y pruebas bioquímicas es un indicador positivo y sensible que evalúa el crecimiento físico y las condiciones de nutrición, identificando oportunamente alteraciones en su salud. El objetivo de este estudio fue identificar el estado nutricional, categorizándolo según el índice de masa corporal (IMC) en desnutrición severa, desnutricion moderada, normal, sobrepeso y obesidad, la presencia de anemia si se presentan valores de Hemoglobina menor de $11,5 \mathrm{~g} / \mathrm{dL}$ y/o Hematocrito menor al 34\% (OMS, 2011) y parásitos de los niños, niñas y adolescentes del Hogar de Amor y Esperanza en la Colonia San José de la Vega de Tegucigalpa en 2017.

\section{MÉTODOS}

El Hogar de Amor y Esperanza es un centro de acogimiento para niños, niñas y adolescentes en situación de vulneración de derechos, con un nivel socioeconómico bajo anualmente se reciben alrededor de 20-25 niños, niñas y adolescentes, con edades comprendidas entre 5 a 19 años, provenientes de diferentes departamentos de nuestro país. Los niños de edades comprendidas entre 5-14 años se encuentran cursando el nivel de educación básica en la escuela "Hogar de Amor y Esperanza", las adolescentes de sexo femenino estudian en el "Instituto Evangélico Virginia Sapp" y los adolescentes de sexo masculino estudian en "Escuela Agrícola de Amor y Esperanza".

Este estudio tiene un enfoque cuantitativo, de diseño no experimental, descriptivo y transversal, se identificó el estado nutricional, la presencia de anemia y de parasitos de los niños, niñas y adolescentes institucionalizados desde por lo menos hace un año en el Hogar de Amor y Esperanza en la Colonia San José de la Vega de Tegucigalpa en 2017, para determinar alteraciones nutricionales y derivarlos a los servicios de salud. 


\section{Aspectos éticos}

A cada participante de acuerdo a su edad se le explicó la naturaleza, propósito y beneficios del estudio garantizándoles la confidencialidad de los datos proporcionados y sus resultados. La participación de los niños se autorizó por medio del consentimiento informado suscrito con el personal responsable de los niños, niñas y adolescentes del Hogar de Amor y Esperanza para participar en la investigación. El estudio se realizó a solicitud de esta institución y aprobado por el Comité de Ética de la Facultad de Ciencias Químicas y Farmacia.

\section{Valoración antropométrica}

Las medidas antropométricas fueron realizadas por las investigadoras de este estudio debidamente entrenadas, se registraron medidas de peso y talla por triplicado del total de la población, obteniéndose la media, utilizando una balanza mecánica con tallímetro" Mechanical Physician Scale Model RL-MPS-20" 0.1Kg/0.2Lb" en la Clínica del Hogar de Amor y Esperanza.

El Índice de masa corporal [IMC=Peso(kg)/Talla2(m2)] se calculó a partir de los datos antropométricos (peso en $\mathrm{Kg}$ y talla en $\mathrm{m}$ ) empleando las tablas de IMC para la edad de los niños y niñas de 5 a 18 años de la OMS (2007) donde clasifica el IMC en 5 categorías: Desnutrición severa $(<-3 \mathrm{SD})$, desnutrición moderada $(\geq-3 a<-2$ SD), Normal ( $\geq-2 \mathrm{a} \leq+1 \mathrm{SD})$, Sobrepeso ( $>+1 \mathrm{a} \leq+2 \mathrm{SD})$ y Obesidad $(>+2 \mathrm{SD})(7)$.

Técnica de medición: Se realizó con el niño(a) o adolescente en ayunas con la vejiga o intestinos vacíos en condiciones óptimas de temperatura. Se utilizó ropa delgada para garantizar un procedimiento adecuado. El niño(a) 0 adolescente estaba de pie, con los talones juntos y el abdomen relajado, los brazos a los lados y el peso repartido en forma equitativa entre ambos pies; se utilizó para la talla el plano de Franckfurt.

La talla y el peso para la edad para los niños, niñas y adolescentes del HAYE se determinaron empleando los Patrones de Crecimiento de la OMS (2007) para las edades de 5 a 19 años. Según la OMS, un percentil denota el porcentaje de la población que siendo normal está por debajo de esa línea, distribuidos en cinco categorías percentil 3, 15, 50, 85 y 97 . Así, si una niña que está en percentil 3 significa que el $3 \%$ de la población de los niños normales tienen un peso inferior a ese. El percentil 3 se emplea como límite de alerta y si se encuentra por debajo de éste se deben realizar estudios para descartar posibles enfermedades causantes (OMS, 2013). 


\section{Pruebas Bioquímicas}

La toma de muestra de sangre y heces para las pruebas bioquímicas la realizó la enfermera especializada del Hogar de Amor y Esperanza y los análisis fueron realizados por microbiólogos en el Laboratorio Microbiológico del Programa del Área de Salud de la Vicerrectoría de Orientación y Asuntos estudiantiles (VOAE) de la UNAH. La población se dividió en cinco grupos de 25 en el transcurso de una semana para la realización de hemograma completo para evaluar si padecen de anemia y examen general de heces para determinar la presencia de parásitos protozoarios y metazoarios en sus diferentes estadios.

El objetivo del examen coproparasitológico fue analizar microscopicamente una muestra de heces en busca de la presencia parásitos protozoarios y metazoarios en sus diferentes estadios. Procedimiento: 1. En una lámina portaobjetos se colocan 2 gotas, en la parte izquierda de solución salina al $0.85 \%$ y en la derecha lugol. 2. Luego se toma con un palillo la muestra de materia fecal (se debe escoger la parte que tenga elementos anormales como sangre, moco, etc. y de otra parte para para que así quede una muestra representativa) 3 . Se homogeniza en la lámina primero en la solución salina y luego en lugol se le colocan los cubreobjetos. 4. La suspensión no debe quedar muy gruesa pero tampoco muy delgada. 5. Observar en forma sistemática al microscopio, con el objetivo 10x luego con 40x y en objetivo de inmersión 100x. Muestra requerida: 5 gramos de heces recién emitidas, sin dejar transcurrir más de 3 horas después de la recolección).

Procedimiento para hemograma: Se recolectaron muestras de sangre venosa de la población infantil en tubos de $5 \mathrm{ml}$ con anticoagulante ácido etilendiaminotetraacético (EDTA). Los hemogramas se realizaron utilizando el equipo automatizado $A B X$ MICROS 60. A aquellas muestras que presentaron alteraciones en los valores hemáticos, se les realizó un frotis sanguíneo teñido con colorante de Wright. Según los valores de referencia bde la Organización Mundial de la Salud (OMS), se definió como anemia un nivel de hemoglobina $<11,5 \mathrm{~g} / \mathrm{dL}$ y/o hematocrito $<34 \%$ en personas $>5$ años (OMS, 2011).

\section{Tratamiento estadístico}

Se realizó el análisis estadístico utilizando el programa informático IBM SPSS 23 (SPSS INC. Chicago, IL., USA), aplicando en todas las pruebas un nivel de significación de 0,05 , y las variables cuantitativas se expresaron en términos de media y desviación estándar. 


\section{RESULTADOS}

El presente estudio fue realizado con la población del Hogar de Amor y Esperanza, de los cuales el $58,97 \% \pm 0,494$ ( 69 niños y adolescentes) son de sexo masculino y el $41,03 \% \pm 0,494$ (48 niñas y adolescentes) son de sexo femenino (Gráfico 1).

Gráfico 1. El sexo de los niños, las niñas y adolescentes del HAYE

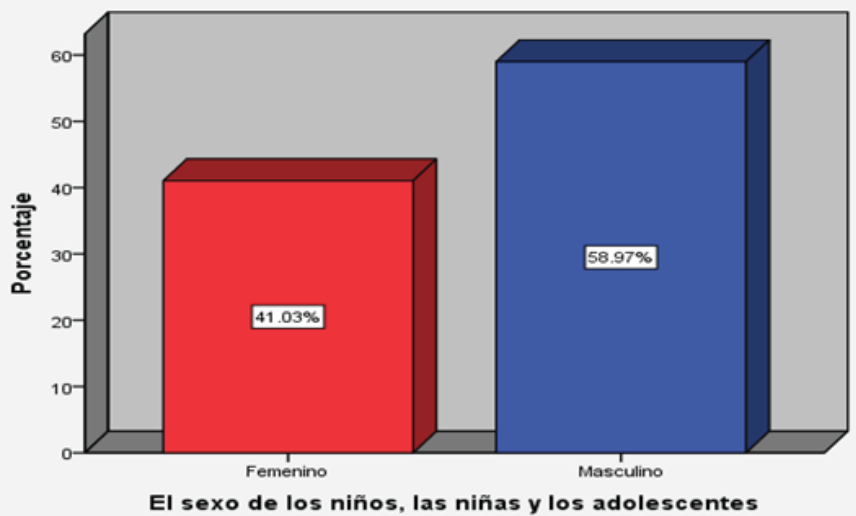

Fuente: Elaboración propia a partir del registro datos generales y antropométricos.

Las edades de los niños, niñas y adolescentes del HAYE están comprendidas entre 5 a 17 años observándose una mayor proporción de niños y niñas en las edades comprendidas de 10 a 12 años de edad, con una desviación estándar de 2,86 y una moda de 12 (Gráfico 2).

Gráfico 2. Distribución de las edades de los niños, niñas y adolescentes del HAYE.

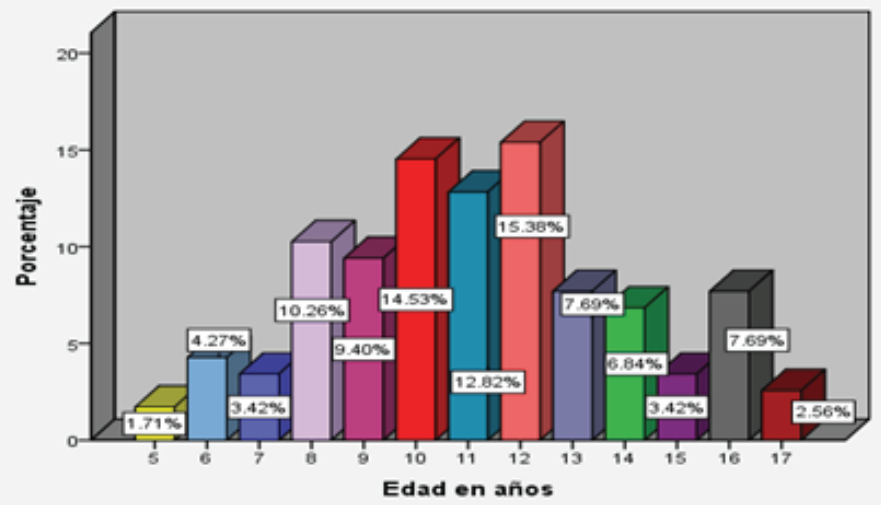

Fuente: Elaboración propia a partir del registro datos generales y antropométricos. 
Según la distribución del IMC de los niños, niñas y adolescentes del HAYE se obtuvo que el 0,85\% (1) presenta una desnutrición severa, el 0,85\% (1) presenta desnutrición moderada, el 94,03\% (110) normal, y el 4,27\% (5) sobrepeso (Tabla 1).

Tabla 1. El índice de masa corporal de los niños, niñas y adolescentes del HAYE.

\begin{tabular}{|l|c|c|}
\multicolumn{1}{|c|}{ IMC } & Número & Porcentaje \\
\hline Desnutrición Severa & 1 & $0.85 \%$ \\
\hline Desnutrición Moderada & 1 & $0.85 \%$ \\
\hline Normal & 110 & $94.03 \%$ \\
\hline Sobrepeso & 5 & $4.27 \%$ \\
\hline Total & 117 & $100.00 \%$ \\
\hline
\end{tabular}

Fuente: Elaboración propia a partir del registro datos antropométricos y tabla de IMC para la edad de los niños de 5 a 18 años de la OMS (2007)

De acuerdo a la talla para la edad de los niños y niñas de 5 a 10 años el 16,0\% (8) se encuentra por debajo del percentil 3, el $26 \%$ (13) en percentil 3 , el $48 \%$ (24) en percentil 15 , el $8 \%$ (4) en percentil 50 y $2 \%$ (1) en percentil 85 observándose un retraso de la talla para edad (Gráfico 3).

Gráfico 3. La talla para la edad de los niños y las niñas de 5 a 10 años de edad

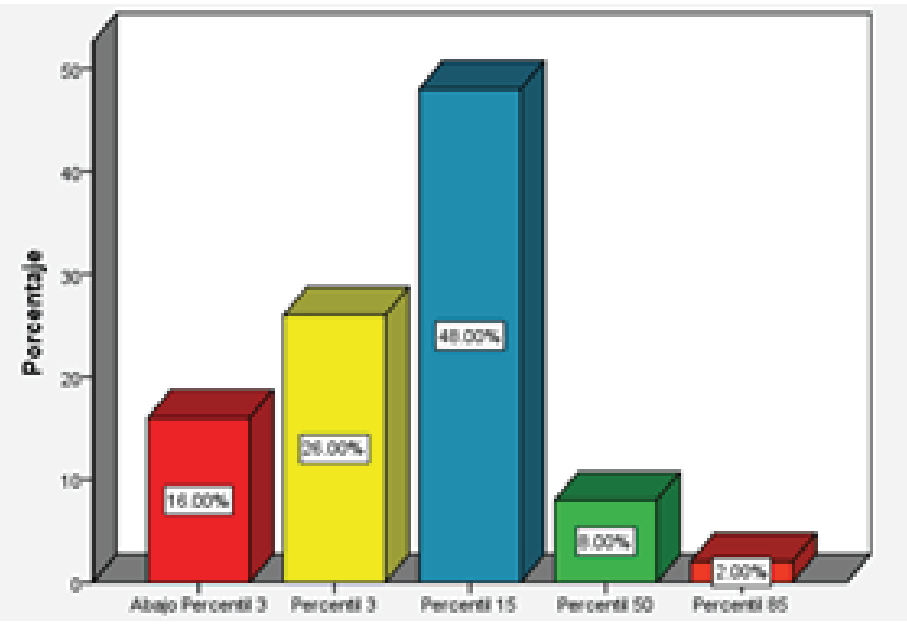

Fuente: Elaboración propia a partir del registro datos antropométricos y tabla de IMC para la edad de los niños de 5 a 18 años de la OMS (2007) 
Gráfico 4. El peso para la edad de los niños y las niñas de 5 a 10 años de edad

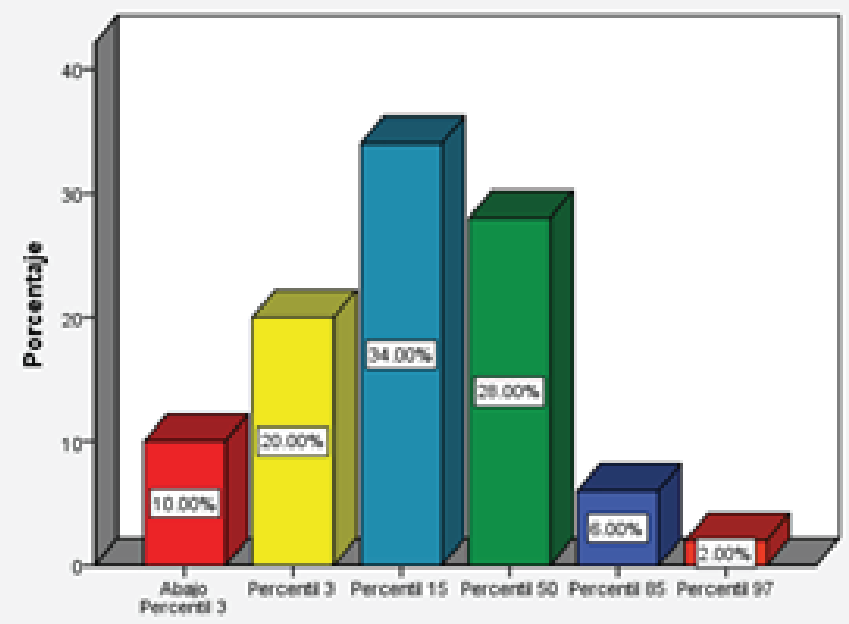

Fuente: Elaboración propia a partir del registro datos antropométricos y tabla de IMC para la edad de los niños de 5 a 18 años de la OMS (2007)

El $77,78 \%$ de los niños, niñas y adolescentes presentan un hemograma normal con valores de Hemoglobina mayores al 11,5 g/dL y/o Hematocrito mayor al 34\% (OMS, 2011), el 5,98\% anormal con valores Hemoglobina menor de 11,5 g/dL y/o Hematocrito menor al 34\% (OMS, 2011) y en el porcentaje restante no se realizó esta prueba (Tabla 2).

Tabla 2. Resultados de hemograma de los niños, niñas y adolescentes del HAYE.

\begin{tabular}{|c|c|c|}
\hline Hemograma & Número & Porcentaje \\
\hline $\begin{array}{l}\text { Hemoglobina }>11,5 \mathrm{~g} / \mathrm{dL} \mathrm{y} / 0 \\
\text { Hematocrito }>34 \%(\mathrm{OMS}, 2011)\end{array}$ & 91 & $77.78 \%$ \\
\hline $\begin{array}{l}\text { Hemoglobina }<11,5 \mathrm{~g} / \mathrm{dL} \text { y/o } \\
\text { Hematocrito }<34 \% \text { (OMS, 2011) }\end{array}$ & $\begin{array}{c}7 \\
10.6-10.7 \\
33.4-33.6\end{array}$ & $5.98 \%$ \\
\hline No se lo realizó & 19 & $16.24 \%$ \\
\hline Total & 117 & $100.00 \%$ \\
\hline
\end{tabular}

Fuente: Elaboración propia a partir del registro de resultados de hemograma y valores de concentraciones de hemoglobina para diagnosticar anemia y evaluar su gravedad. OMS (2011). 
Los niños presentaron alto porcentaje de parasitismo $80.56 \%$ (58) y las especies prevalentes fueron Blastocystis hominis $38.89 \%$ (28), Endolimax nana quiste $37.5 \%$ (27), Entamoeba coli quiste (25) lodamoeba Butschlii quiste 22.22\% (16). De los exámenes de heces realizados se observó la presencia de 1 parásito en $27.78 \%(20)$, presencia de 2 parásitos $31.94 \%$ (23), presencia de 3 parásitos $20.83 \%$ (15) y no se observaron parásitos en el $19.44 \%$ (14) de los niños y adolescentes del HAYE (Gráfico 5).

\section{Gráfico 5. Prevalencia de parásitos de los niños, las niñas y adolescentes del HAYE}

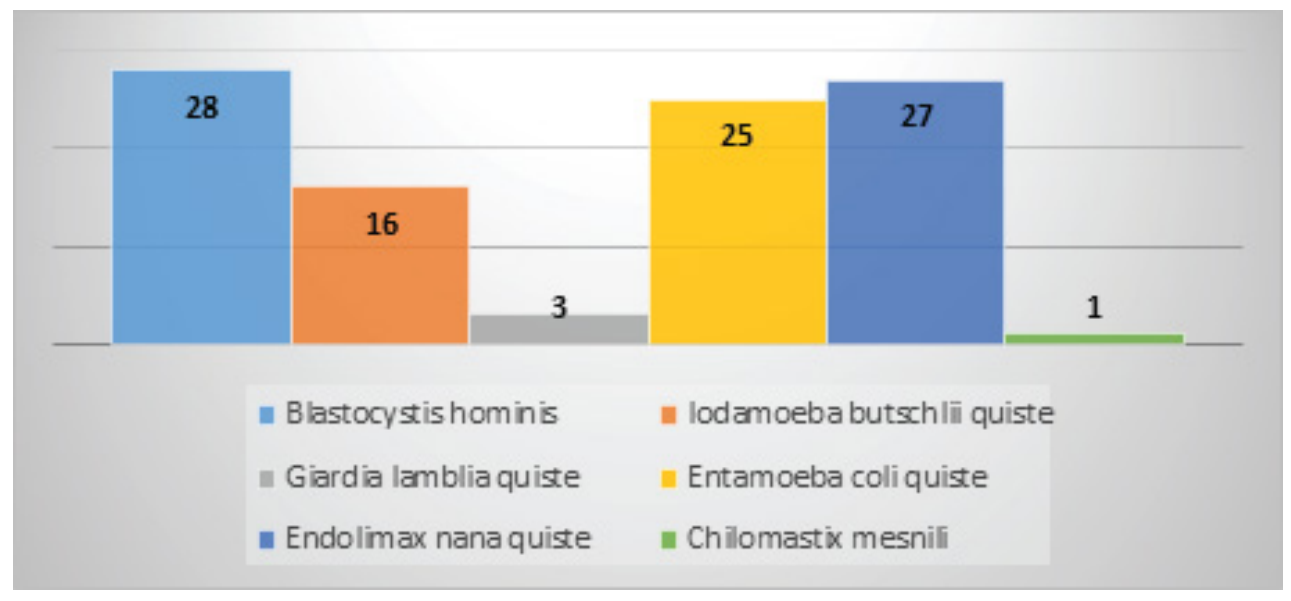

Fuente: Elaboración propia a partir del registro de resultados de examen coproparasitológico.

\section{DISCUSIÓN}

En el estudio actual según la distribución del IMC de los niños, niñas y adolescentes del HAYE, el $0.85 \%$ presentó desnutrición severa, el $0.85 \%$ desnutrición moderada y $4.27 \%$ de sobrepeso, mientras que en el estudio "Prevalencia de sobrepeso y obesidad en niños de 6 a 12 años de tres Escuelas de Honduras" en el 2016, de Medina, Yuja y Lanza (10) obtuvieron que la prevalencia de sobrepeso fue de un $11.8 \%$, de obesidad de un $15.6 \%$, el $5.7 \%$ presentó bajo peso y el resto normal, similar al estudio "Caracterización clínico-antropométrica y estado nutricional en escolares de 6-11 años" realizado en el Hospital Pediátrico Universitario "Pedro Soto Alba" en Holguín, Cuba, se encontró que el 73,1 \% de los niños fue normopeso, 8,6 \% obesos, 10,5\% sobrepesos y solo 3,6 \% desnutridos (Torres, 2016). 
En el presente estudio el $0.85 \%$ presentó desnutrición severa, el $0.85 \%$ desnutrición moderada y sobrepeso el $4.27 \%$ mientras que en el estudio Estado Nutricional en niños de 6 a 10 años de edad de la comunidad infantil "Sagrada Familia" en Lima, Perú (Carrasco et al., 2011) el 5.6\% de niños presentaron desnutrición global, 23.8\% desnutrición crónica, $21.4 \%$ sobrepeso y $2.4 \%$ obesidad.

Se realizó un estudio de prevalencia poblacional: Estado Nutricional en Escolares del Valle del Cauca (Munevar et al., 2012), con una población 1789 escolares seleccionados aleatoriamente con el indicador talla para la edad, se identificó retraso en talla en $8,9 \%$, riesgo de talla baja en $25,1 \%$ y en el presente estudio se obtuvo un retraso en la talla en $42 \%$ y en riesgo de talla baja en $48 \%$.

Se observó un elevado porcentaje de adolescentes que presentaron un riesgo nutricional elevado (35\%), resultados que muestran una realidad preocupante en el estudio "Evaluación del riesgo nutricional de los adolescentes escolarizados en Cantabria" de (De-Rufino et al.,2014) en un muestra de 1101 adolescentes de edades comprendidas entre los 10 y los 17 años durante el 2011 mientras que en el presente estudio se obtuvo un bajo porcentaje de riesgo nutricional en los niños, niñas y adolescentes del HAYE.

De acuerdo a la talla para la edad de los niños y niñas de 5 a 10 años del HAYE el $16,0 \%$ se encuentra por debajo del percentil 3 , el $26 \%$ en percentil 3 , y el $48 \%$ en percentil 15 , observándose un elevado retraso de la talla para la edad, a diferencia del estudio "Estado nutricional en niños escolares. Valoración clínica, antropométrica y alimentaria publicada en la Revista Cientíica de las Ciencias Médicas en Cienfuegos, Cuba en 2010, donde se encontró que ningún niño presentó talla por debajo del tercer percentil (González et al., 2010).

Al igual que el estudio "Evaluación de la calidad de vida en escolares con antecedentes de desnutrición temprana severa" realizado con niños de 5-12 años de edad en Argentina el 2014 por E.S. De Grandis, P.A. Armelini y E. Cuestas, concluimos que la desnutrición en niños pequeños continúa siendo un problema grave de salud en el mundo al que debemos estar vigilante. Las secuelas sobre el sistema nervioso central condicionan un retraso en la adquisición de habilidades, dificultades de aprendizaje, menor capacidad de adaptación y pérdida de oportunidades de educación y laborales. Por ende, a largo plazo los niños desnutridos no tendrán las mismas oportunidades de trabajo; en el adulto se afectará la productividad y el capital humano (De Grandis, Armelini y Cuestas, 2014). 
Se observó correlación entre el estado nutricional actual, los índices antropométricos evaluados y las pruebas bioquímicas.

\section{CONCLUSIONES}

Los niños, niñas y adolescentes del Hogar de Amor y Esperanza presentan un buen estado nutricional de acuerdo a los parámetros de IMC dados por la OMS (2007), examen hematológico y de heces según las referencias del laboratorio de la UNAH. Hay mayor prevalencia de retraso en la talla que en el peso de los niños, niñas y adolescentes del Hogar de Amor y Esperanza según tablas de referencia de la OMS (2007).

En los niños, niñas y adolescentes del Hogar de Amor y Esperanza se presenta baja incidencia de anemia.

Existe una gran prevalencia de parásitos intestinales protozoarios y metazoarios en los niños, niñas y adolescentes del Hogar de Amor y Esperanza, donde predominan el Blastocystis hominis, la Entamoeba coli y la Giardia lamblia, lo cual puede ser a consecuencia de los hábitos de higiene inadecuados que los niños tienen en el ámbito social del cual provienen, lo que favorece los procesos continuos de infestación por protozoarios y helmintos intestinales.

Se recomienda dar un seguimiento habitual del estado nutricional, implementar programas de educación nutricional para fortalecer el consumo de alimentos que garanticen a los niños, niñas y adolescentes del Hogar de Amor y Esperanza una nutrición adecuada y prestar una mayor atención a aquellos que presentaron bajo peso, retraso en la talla, anemia y parasitosis intestinal.

\section{AGRADECIMIENTOS}

Agradecimientos especiales a las autoridades de la Carrera de Química y Farmacia, del Programa de Salud de la Vicerrectoría de Orientación y Asuntos Estudiantiles de la UNAH y a la Dirección de Investigación Científica y Postgrado para la realización de este estudio. 


\section{REFERENCIAS BIBLIOGRÁFICAS}

Carrasco Rueda, José et al. (2012). Estado Nutricional en niños de 6 a 10 años de edad de la comunidad infantil "Sagrada Familia". Lima, marzo 2011. Horizonte Médico, [S.I.], v. 12, n. 1, p. 27-31. Disponible en: <http://www.horizontemedicina.usmp.edu.pe/index.php/horizontemed/article/view/93

De Grandis E.S, Armelini P.A, Cuestas E. (2014). Evaluación de la calidad de vida en escolares con antecedentes de desnutrición temprana severa. An.Pediatr (Barc) 81(6):368-373. Disponible en:http://www.analesdepediatria.org/es/evaluacion-calidad-vida-escolares-con/articulo/S1695403313004888/

De-Rufino Rivas PM, Antolín Guerra O, Casuso Ruíz I, Amigo Lanza T, Noriega Borge MJ, Santamaría Pablos A, "et-al". (2014) Evaluación del riesgo nutricional de los adolescentes escolarizados en Cantabria. Nutr Hosp. 29(3):652-657. Disponible en: http://scielo.isciii.es/scielo.php?script=sci_arttext\&pid=S021216112014003300226\&lng=es. http://dx.doi.org/10.3305/NH.2014.29.3.7190

FANTA-BMI-charts (2013). Tablas de IMC para la edad, de niños(as) y adolescentes de 5 a 18 años de edad y tablas de IMC para adultos(as) no embarazadas, no lactantes $\otimes 19$ años de edad. Disponible en: http://www.fantaproject.org/sites/default/files/resources/FANTA-BMI-charts-Jan2013-ENG_0.pdf

Fariñas-Rodríguez L, Vázquez-Sánchez V, Martínez-Fuentes AJ, Carmenate-Moreno MM, Marrodán MD. (2011). Evaluación del estado nutricional de escolares cubanos y españoles: índice de masa corporal frente a porcentaje de grasa. Nutr. Clín. Diet. Hosp.32(2):58-64.Disponible en: http://www.nutricion.org/publicaciones/revista_2012_ 32_2/EVALUACION-ESTADO.pdf

FUNIBER (2011) Nutrición Infantil y Origen Fetal de las Patologías. Capítulo 4. Nutrición del Adolescente. 1.13 Métodos de valoración del crecimiento. Estudio antropométrico. Madrid: PANAMERICANA; p. 73. Fondo de las Naciones Unidas para la Infancia UNICEF (2013). Informes sobre el Estado Mundial de la Infancia. Disponible en: http://www.unicef.org/spanish/sowc/index_38236.html

González Hermida A E, Vila Díaz J, Guerra Cabrera CE, Quintero Rodríguez O, Dorta Figueredo M, Pacheco JD. (2010). Estado nutricional en niños escolares. Valoración clínica, antropométrica y alimentaria. MediSur 8(2):15-22. Disponible en: http://scielo.sld.cu/pd$\mathrm{f} / \mathrm{ms} / \mathrm{v} 8 \mathrm{n} 2 / \mathrm{v} 8 \mathrm{n} 2 \mathrm{a} 864$.pdf

Mataix Verdú J, López Jurado Romero de la Cruz M. (2009) Evaluación de estado nutricional.

En Mataix Verdú J, editor. Nutrición y alimentación. Vol. 2. Barcelona: OCÉANO/ergon; p.958-959.

Martínez Hernández A, Portillo Baquedano MP. (2011) Nutrición y estado nutricional. En Martínez, Portillo. Fundamentos de nutrición y dietética. Bases metodológicas y aplicaciones. Madrid: PANAMERICANA; p.77. Disponible en: http://www.medicapanamericana.com/VisorEbookV2/Ebook/

Medina Acosta C, Yuja Suárez N, Lanza Martínez O. (2016). Prevalencia de sobrepeso y 
obesidad en niños de 6 a 12 años de tres Escuelas de Honduras. Arch de Medi. 12(3). Disponible en: http://www.archivosdemedicina.com/medicina-de-familia/prevalencia-desobrepeso-y-obesidad-en-nintildeos-de-6-a-12-antildeos-de-tres-escuelas-de-honduras php?aid=11260oi:10.3823/1312

Munevar Cubides AM, Moreno Casallas FO, Guarín Salazar J, Barros González GI, Villamarín Betancourt EA, Hernández Carrillo M. (2012). Estado Nutricional en Escolares del Valle del Cauca. Revista Colombiana Salud Libre 9 (2): 84-90. Disponible en: http://revistasojs.unilibrecali.edu.co/index.php/rcslibre/article/view/25

Organización Mundial de la Salud. (2011). Concentraciones de hemoglobina para diagnosticar anemia y evaluar su gravedad. Ginebra, Suiza. Disponible en: http://www.who.intiris/handle/10665/85842

Organización Mundial de la Salud. (2016) Patrones del crecimiento infantil. Ginebra. Disponible en: http://www.who.int/mediacentre/factsheets/fs355/es/

Torres Molina A. (2011) Caracterización clínico-antropométrica y estado nutricional en escolares de 6-11 años. Medisur 9(3): 215-222. Disponible en: http://scielo.sld.cu/scielo.php?script=sci_arttext\&pid=S1727-897X2011000300004\&lng=es. 